\title{
Analytical Solutions for Sequentially Reactive Transport with Different Retardation Factors
}

Y. Sun, T.A. Buscheck, K. Mansoor, X. Lu

August 1, 2001

U.S. Department of Energy

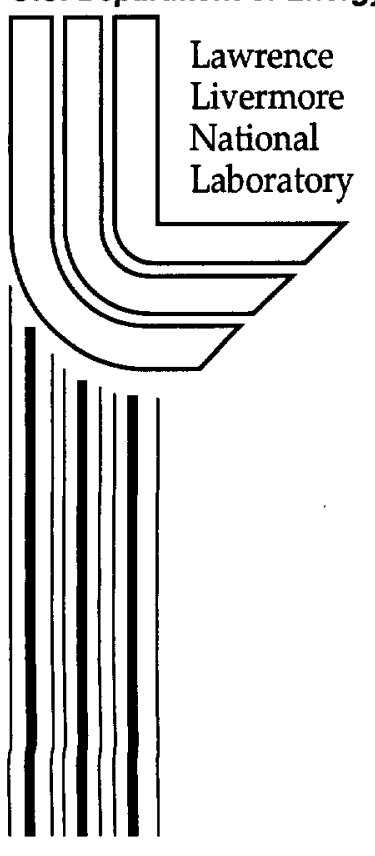




\section{DISCLAIMER}

This document was prepared as an account of work sponsored by an agency of the United States Government. Neither the United States Government nor the University of California nor any of their employees, makes any warranty, express or implied, or assumes any legal liability or responsibility for the accuracy, completeness, or usefulness of any information, apparatus, product, or process disclosed, or represents that its use would not infringe privately owned rights. Reference herein to any specific commercial product, process, or service by trade name, trademark, manufacturer, or otherwise, does not necessarily constitute or imply its endorsement, recommendation, or favoring by the United States Government or the University of California. The views and opinions of authors expressed herein do not necessarily state or reflect those of the United States Government or the University of California, and shall not be used for advertising or product endorsement purposes.

This is a preprint of a paper intended for publication in a journal or proceedings. Since changes may be made before publication, this preprint is made available with the understanding that it will not be cited or reproduced without the permission of the author.

This report has been reproduced directly from the best available copy.

Available electronically at http://www.doe.gov/bridge

Available for a processing fee to U.S. Department of Energy

and its contractors in paper from

U.S. Department of Energy

Office of Scientific and Technical Information

P.O. Box 62

Oak Ridge, TN 37831-0062

Telephone: (865) 576-8401

Facsimile: (865) 576-5728

E-mail: reports@adonis.osti.gov

Available for the sale to the public from

U.S. Department of Commerce

National Technical Information Service

5285 Port Royal Road

Springfield, VA 22161

Telephone: (800) 553-6847

Facsimile: (703) 605-6900

E-mail: orders@ntis.fedworld.gov

Online ordering: http://www.ntis.gov/ordering.htm

OR

Lawrence Livermore National Laboratory

Technical Information Department's Digital Library

http://www.llnl.gov/tid/Library.html 


\title{
Analytical Solutions for Sequentially Reactive Transport with Different Retardation Factors
}

\author{
Yunwei Sun ${ }^{1}$, Thomas A. Buscheck ${ }^{1}$, Kayyum Mansoor ${ }^{2}$, Xinjian Lu ${ }^{3}$ \\ ${ }^{1}$ Lawrence Livermore National Laboratory, Livermore \\ ${ }^{2}$ Weiss Associates, Emeryville \\ ${ }^{3}$ California State University; Hayward
}

\begin{abstract}
Integral transforms have been widely used for deriving analytical solutions for solute transport systems. Often, analytical solutions can only be written in closed form in frequency domains and numerical inverse-transforms have to be involved to obtain semi-analytical solutions in the time domain. For this reason, previously published closed form solutions are restricted either to a small number of species or to the same retardation assumption. In this paper, we applied the solution scheme proposed by Bauer et al. [2001] in the time domain. Using available analytical solutions of a single species transport with first-order decay without coupling with its parent species concentration as fundamental solutions, a daughter species concentration can be expressed as a linear function of those fundamental solutions. The implementation of the solution scheme is straight forward and exact analytical solutions are derived for one- and three-dimensional transport systems.
\end{abstract}

Key words: Analytical solution, retardation factor, multispecies reactive transport, first-order reaction.

\section{Introduction}

The analytical solution of Sun et al. [1999] to sequentially reactive transport has been used as a screening tool for evaluating groundwater contamination and simulating natural attenuation [Aziz et $a l ., 1999]$. However, the solution is limited to cases where retardation factors of all species are equal. There is a need to develop analytical solutions to the system with different retardation factors to accurately simulate natural conditions.

Derivation of analytical solutions of solute transport system usually involves complex mathematical manipulation in order to convert solutions from a frequency domain to a time domain. For this reason, previously published analytical solutions to the transport of first-order decay chains are limited to a small number of species [van Genuchten, 1985; Lunn et al., 1996]. Although Sun et al. [1999] extended analytical solutions to N-species, retardation factors were not incorporated. Eykholt and $\mathrm{Li}$ [2000] developed a semi-analytical solution of a linear reaction network using a response function approach. Since numerical convolution is involved, it is difficult to implement the approach as a screening tool. Recently, Bauer et al. [2001] developed a Laplace domain solution using a recursive form. The concentration of a daughter species is expressed as a linear function of ancestor concentrations and the factor of each species concentration is calculated using the recursive form. Though Bauer et al. [2001] has made significant progress in first-order reactive transport, the complexity of 
inverse Laplace transform makes the code implementation difficult. When numerical inverse Laplace transforms are involved, the approach becomes even more complicated. Both Eykholt and Li [2000] and Bauer et al. [2001] are based on the unimolar assumption, that is, the stoichiometry of the reaction is such that $1 \mathrm{~mol}$ of product is produced by consuming $1 \mathrm{~mol}$ of reactant.

In order to avoid the difficulty with inverse Laplace transforms and with numerical processes, we propose an approach to develop closed form solutions of first-order reactive chains in the time domain. We also incorporate yield coefficient factors to the solution approach and analytical solutions. For the sake of simplicity, we demonstrate the solution scheme for a one-dimensional solution to a fourspecies reactive problem. The solution derived in this paper is compared with Lunn et al. [1996] and Sun et al. [1999]. Further, we extended the one-dimensional solutions to a three-dimensional system.

\section{First-order reactive transport}

The transport of a first-order decay chain can be written as [Bear, 1979]:

$$
\mathcal{L}\left(c_{i}\right)=R_{i} \frac{\partial c_{i}}{\partial t}+R_{i} k_{i} c_{i}-R_{i-1} y_{i-1} k_{i-1} c_{i-1}, \quad \forall i=1,2, \ldots, n
$$

where $\mathcal{L}$ is the advective-dispersive operator, which is linear in $c_{i}$ and can be simplified in a homogenous one-dimensional column as

$$
\mathcal{L}=D \frac{\partial^{2}}{\partial x^{2}}-v \frac{\partial}{\partial x}
$$

where $v$ is the constant flow velocity $\left[L T^{-1}\right]$ and $D$ represents a constant hydrodynamic dispersion coefficient $\left[L^{2} T^{-1}\right]$ :

$$
D=a v+\mathcal{D}
$$

where $a$ is dispersivity $[L], \mathcal{D}$ is the molecular diffusion coefficient $\left[L^{2} T^{-1}\right]$, and $R_{i}$ and $k_{i}$ represent the retardation factor and the first-order decay rate of species $i$, respectively. $y_{i-1}$ is the stoichiometrical yield factor calculated as the concentration ratio of $c_{i}$ to $c_{i-1}$ in the reaction from species $i-1$ to species $i$. Eq. (1) differs from the basic equation of Sun et al. [1999] by the species-specific retardation factors. The transform of Sun et al. [1999] fails to derive analytical solutions when $R_{i} \neq R_{j}$. D is assumed to be negligible, thus, $D=a v$ is used in the rest of this paper.

\section{Solution method}

Since equation (1) for species $i$ is linear in $c_{i}$, the solution of $c_{i}$ can be written as a linear combination of the fundamental solutions $\Omega_{j} \quad \forall j=1,2, \cdots, n$

$$
c_{i}=\sum_{j=1}^{i} A_{j}^{i} \Omega_{j}
$$

or in matrix format

$$
\mathbf{c}=\mathbf{A} \Omega,
$$

where $\Omega_{i}, \forall i=1, \cdots, n$, represents the analytical solution of $i$ th species concentration in the time domain without coupling with the concentration of its parent species, $c_{i-1}$,

$$
\mathcal{L}\left(\Omega_{i}\right)=R_{i} \frac{\partial \Omega_{i}}{\partial t}+R_{i} k_{i} \Omega_{i}, \quad \forall i=1,2, \ldots, n .
$$


$\mathbf{A}$ is called transform matrix and $A_{j}^{i}=0 \forall j>i$. If all components of $\mathbf{A}, A_{j}^{i}, \forall j<i, i=1,2, \cdots, n$, can be expressed as closed form functions of system parameters and fundamental solutions, $\Omega_{j}, j=$ $1,2, \cdots, i$, the analytical solutions of $\mathbf{c}$ in (5) become available.

Similarly to Bauer et al. [2001], by substituting (4) into (1),

$$
\sum_{j=1}^{i} A_{j}^{i}\left[\left(R_{i}-R_{j}\right) \frac{\partial \Omega_{j}}{\partial t}+\left(R_{i} k_{i}-R_{j} k_{j}\right) \Omega_{j}\right]=\sum_{j=1}^{i-1} A_{j}^{i-1} R_{i-1} y_{i-1} k_{i-1} \Omega_{j}
$$

Since the last term on the left hand of equation ( 7 ) equals zero when $j=i$,

$$
\sum_{j=1}^{i-1} A_{j}^{i}\left[\left(R_{i}-R_{j}\right) \frac{\partial \Omega_{j}}{\partial t}+\left(R_{i} k_{i}-R_{j} k_{j}\right) \Omega_{j}\right]=\sum_{j=1}^{i-1} A_{j}^{i-1} R_{i-1} y_{i-1} k_{i-1} \Omega_{j}
$$

Equation (8) holds, if each term on the left equals the corresponding term on the right,

$$
A_{j}^{i}\left[\left(R_{i}-R_{j}\right) \frac{\partial \Omega_{j}}{\partial t}+\left(R_{i} k_{i}-R_{j} k_{j}\right) \Omega_{j}\right]=A_{j}^{i-1} R_{i-1} y_{i-1} k_{i-1} \Omega_{j} \quad \forall j=1,2, \cdots, i-1 .
$$

Then, the components of the transform matrix can be written in a recursive format

$$
A_{j}^{i}=A_{j}^{i-1} \frac{R_{i-1} y_{i-1} k_{i-1} \Omega_{j}}{\left(R_{i}-R_{j}\right) \frac{\partial \Omega_{j}}{\partial \iota}+\left(R_{i} k_{i}-R_{j} k_{j}\right) \Omega_{j}} .
$$

If $R_{i}=R_{j}$

$$
A_{j}^{i}=A_{j}^{i-1} \frac{y_{i-1} k_{i-1}}{k_{i}-k_{j}}
$$

the transform format of Sun et al. [1999] can be derived. Therefore, the approach Sun et al. [1999] proposed is a typical case of $(10)$.

\section{Solution Implementation}

The analytical solution of Bear $[1979$, p. 268] to a single species transport with first-order decay in a semi-infinite column is written as:

$$
f=\frac{c_{o}}{2} \exp (\alpha x) \operatorname{erfc}(\beta)
$$

where

$$
\alpha=\frac{1}{2 a}-\left(\frac{1}{4 a^{2}}+\frac{k}{D}\right)^{1 / 2} \quad \beta=\frac{x-\left(v^{2}+4 k D\right)^{1 / 2} t}{2(D t)^{1 / 2}} \quad D=a v .
$$

Let

$$
\Omega_{i}=\frac{1}{2} \exp \left(\alpha_{i} x\right) \operatorname{erfc}\left(\beta_{i}\right) \quad \forall i=1,2, \cdots, n .
$$

Correspondingly,

$$
\alpha_{i}=\frac{1}{2 a}-\left(\frac{1}{4 a^{2}}+\frac{k_{i}}{D_{i}}\right)^{1 / 2} \quad \beta_{i}=\frac{x-\left(v_{i}^{2}+4 k_{i} D_{i}\right)^{1 / 2} t}{2\left(D_{i} t\right)^{1 / 2}} \quad D_{i}=a v_{i}
$$


where $v_{i}=v / R_{i}$ is the transport velocity of species $i$. When $x=0$,

$$
\left.\Omega_{i}\right|_{x=0}=1, \quad \forall i=1,2, \cdots, n .
$$

When $\beta \gg 2, \Omega$ function reaches steady state

$$
\Omega_{i}=\exp \left(\alpha_{i} x\right) \quad \forall \beta_{i} \gg 2, \quad i=1,2, \cdots, n .
$$

Then, the first derivative of $\Omega_{i}$ in terms of time can be written as

$$
\frac{\partial \Omega_{i}}{\partial t}=\frac{1}{4 \sqrt{D_{i} \pi}} \exp \left(\alpha_{i} x-\beta_{i}^{2}\right)\left[\frac{x}{t^{3 / 2}}+\sqrt{\frac{v_{i}^{2}+4 k_{i} D_{i}}{t}}\right] \quad \forall i=1,2, \cdots, n .
$$

To implement this solution scheme, we take a transport of four sequentially reactive species as an example. According to (4),

$$
\left\{\begin{array}{l}
c_{1}=A_{1}^{1} \Omega_{1} \\
c_{2}=A_{1}^{2} \Omega_{1}+A_{2}^{2} \Omega_{2} \\
c_{3}=A_{1}^{3} \Omega_{1}+A_{2}^{3} \Omega_{2}+A_{3}^{3} \Omega_{3} \\
c_{4}=A_{1}^{4} \Omega_{1}+A_{2}^{4} \Omega_{2}+A_{3}^{4} \Omega_{3}+A_{4}^{4} \Omega_{4} .
\end{array}\right.
$$

If the boundary condition is defined as

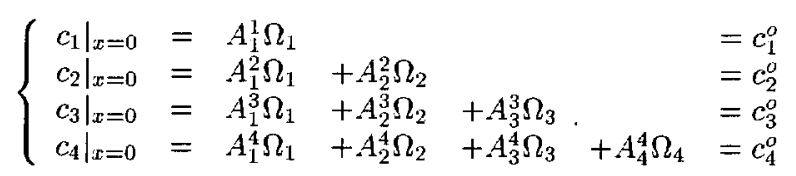

the components of the transform matrix $\mathbf{A}$ can be derived successively as

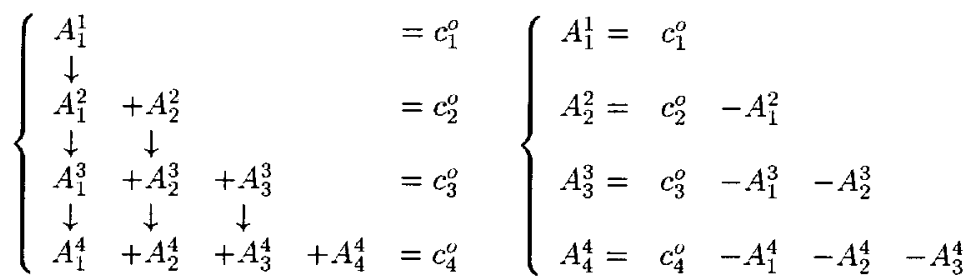

$$
\begin{aligned}
& A_{1}^{2}=A_{1}^{1} \frac{R_{1} y_{1} k_{1} \Omega_{1}}{\left(R_{2}-R_{1}\right) \frac{\partial \Omega_{1}}{\partial t}+\left(R_{2} k_{2}-R_{1} k_{1}\right) \Omega_{1}} \quad A_{1}^{3}=A_{1}^{2} \frac{R_{2} y_{2} k_{2} \Omega_{1}}{\left(R_{3}-R_{1}\right) \frac{\partial \Omega_{1}}{\partial t}+\left(R_{3} k_{3}-R_{1} k_{1}\right) \Omega_{1}} \\
& A_{2}^{3}=A_{2}^{2} \frac{R_{2} y_{2} k_{2} \Omega_{2}}{\left(R_{3}-R_{2}\right) \frac{\partial \Omega_{2}}{\partial t}+\left(R_{3} k_{3}-R_{2} k_{2}\right) \Omega_{2}} \quad A_{1}^{4}=A_{1}^{3} \frac{R_{3} y_{3} k_{3} \Omega_{1}}{\left(R_{4}-R_{1}\right) \frac{\partial \Omega_{1}}{\partial t}+\left(R_{4} k_{4}-R_{1} k_{1}\right) \Omega_{1}} \\
& A_{2}^{4}=A_{2}^{3} \frac{R_{3} y_{3} k_{3} \Omega_{2}}{\left(R_{4}-R_{2}\right) \frac{\partial \Omega_{2}}{\partial t}+\left(R_{4} k_{4}-R_{2} k_{2}\right) \Omega_{2}} \quad A_{3}^{4}=A_{3}^{3} \frac{R_{3} y_{3} k_{3} \Omega_{3}}{\left(R_{4}-R_{3}\right) \frac{\partial \Omega_{3}}{\partial t}+\left(R_{4} k_{4}-R_{3} k_{3}\right) \Omega_{3}} .
\end{aligned}
$$

Since $\Omega_{j}$ and $\partial \Omega_{j} / \partial t$ have closed analytical formulae (13) and (16), respectively, the transform matrix $\mathbf{A}$ is exactly analytical and the concentration solutions are analytically described using (5). Though the analytical solution is not written in a single formula, the implementation of the solution scheme can be summarized as

$$
\mathbf{\Omega} \longrightarrow \frac{d \Omega}{d t} \longrightarrow \mathbf{A} \longrightarrow \mathbf{c}
$$




\section{Application and Analysis}

\subsection{Comparison with Lunn et al. [1996]}

Lunn et al. [1996] developed analytical solution to a transport system of three species first-order decay chain. The model is written as

$$
\begin{gathered}
\left(1+K_{d}\right) \frac{\partial c_{1}}{\partial t}=D \frac{\partial^{2} c_{1}}{\partial x^{2}}-v \frac{\partial c_{1}}{\partial x}-k_{1}^{\prime} c_{1} \\
\frac{\partial c_{2}}{\partial t}=D \frac{\partial^{2} c_{2}}{\partial x^{2}}-v \frac{\partial c_{2}}{\partial x}-k_{2} c_{2}+k_{1}^{\prime} c_{1} \\
\frac{\partial c_{3}}{\partial t}=D \frac{\partial^{2} c_{3}}{\partial x^{2}}-v \frac{\partial c_{3}}{\partial x}-k_{3} c_{3}+k_{2} c_{2}
\end{gathered}
$$

where $k_{1}^{\prime}=k_{1}\left(1+K_{d}\right)$ and $K_{d}$ is constant adsorption coefficient. We used the same column geometry and defined system parameters as shown in Table 1.

Table 1. System parameters used in Lumn et al.'s [1996] model

\begin{tabular}{llll}
\hline Velocity & $v$ & 0.1 & $\mathrm{~cm} \mathrm{~h}^{-1}$ \\
Dispersion coefficient & $D$ & 0.18 & $\mathrm{~cm}^{2} h^{-1}$ \\
Retardation factor 1 & $R_{1}=1+K_{d}$ & 2 & \\
Retardation factor 2 & $R_{2}$ & 1 & \\
Retardation factor 3 & $R_{3}$ & 1 & \\
1st-order reaction rate 1 & $k_{1}$ & 0.025 & $h^{-1}$ \\
1st-order reaction rate 2 & $k_{2}$ & 0.03 & $h^{-1}$ \\
1st-order reaction rate 3 & $k_{3}$ & 0.02 & $h^{-1}$ \\
\hline
\end{tabular}

The initial and boundary conditions are assumed as

$$
\begin{aligned}
& c_{1}(x, 0)=c_{2}(x, 0)=c_{3}(x, 0)=0 \\
& c_{1}(0, t)=1.0 \quad c_{2}(0, t)=c_{3}(0, t)=0
\end{aligned}
$$

Figure 1 demonstrates a good match between the solution derived by Lunn et al. [1996] and the new solution derived in this paper after $200 \mathrm{~h}$. Although the new solution can be considered identical to Lunn et al.'s solution, the coding effort required is significantly reduced. 


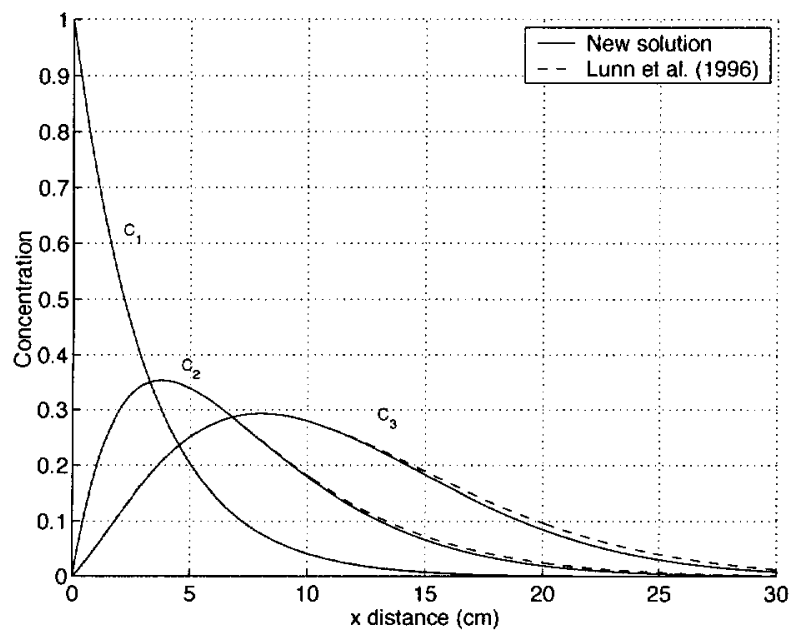

Figure 1. Concentration profiles of three species transport with a constant boundary condition. $t=200 h, R=(2,1,1), k=(0.025,0.03,0.02) h^{-1}, c_{0}=(1.0,0.0,0.0), a=1.8 \mathrm{~cm}^{2} h^{-1}$, $v=0.1 \mathrm{cmh}^{-1}$.

\subsection{Comparison with Sun et al. [1999]}

In order to demonstrate that the Sun et al. [1999] transform is a typical case in (10), we use a four-species transport problem in a one-dimensional column [Bear, 1979, p. 268] and assume $\mathbf{c}^{o}=[1,0,0,0], R_{1}=R_{2}=R_{3}=R_{4}=1$. Using the Sun et al. [1999] transform, the solution of four species concentrations can be written

$$
\begin{aligned}
& c_{1}=a_{1} \\
& c_{2}=a_{2}-\frac{y_{1} k_{1}}{k_{1}-k_{2}} c_{1} \\
& c_{3}=a_{3}-\frac{y_{2} k_{2}}{k_{2}-k_{3}} c_{2}-\frac{y_{1} y_{2} k_{1} k_{2}}{\left(k_{1}-k_{3}\right)\left(k_{2}-k_{3}\right)} c_{1} \\
& c_{4}=a_{4}-\frac{y_{3} k_{3}}{k_{3}-k_{4}} c_{3}-\frac{y_{2} y_{3} k_{2} k_{3}}{\left(k_{2}-k_{4}\right)\left(k_{3}-k_{4}\right)} c_{2}-\frac{y_{1} y_{2} y_{3} k_{1} k_{2} k_{3}}{\left(k_{1}-k_{4}\right)\left(k_{2}-k_{4}\right)\left(k_{3}-k_{4}\right)} c_{1}
\end{aligned}
$$

where

$$
\begin{aligned}
& a_{1}=\Omega_{1} \\
& a_{2}=\frac{y_{1} k_{1}}{k_{1}-k_{2}} \Omega_{2} \\
& a_{3}=\frac{y_{1} y_{2} k_{1} k_{2}}{\left(k_{1}-k_{3}\right)\left(k_{2}-k_{3}\right)} \Omega_{3} \\
& a_{4}=\frac{y_{1} y_{2} y_{3} k_{1} k_{2} k_{3}}{\left(k_{1}-k_{4}\right)\left(k_{2}-k_{4}\right)\left(k_{3}-k_{4}\right)} \Omega_{4} .
\end{aligned}
$$

Substituting $a_{i}, \forall i=1,2,3,4$ and the ancestor concentrations in the daughter species concentrations, the species concentrations can be expressed as linear functions of the basic solutions (4)

$$
c_{1}=\Omega_{1}
$$




$$
\begin{aligned}
c_{2}= & -\frac{y_{1} k_{1}}{k_{1}-k_{2}} \Omega_{1}+\frac{y_{1} k_{1}}{k_{1}-k_{2}} \Omega_{2} \\
c_{3}= & \frac{y_{1} y_{2} k_{1} k_{2}}{{ }_{0}\left(k_{1}-k_{2}\right)\left(k_{1}-k_{3}\right)} \Omega_{1}-\frac{y_{1} y_{2} k_{1} k_{2}}{\left(k_{1}-k_{2}\right)\left(k_{2}-k_{3}\right)} \Omega_{2}+\frac{y_{1} y_{2} k_{1} k_{2}}{\left(k_{1}-k_{3}\right)\left(k_{2}-k_{3}\right)} \Omega_{3} \\
c_{4}= & -\frac{y_{1} y_{2} y_{3} k_{1} k_{2} k_{3}}{\left(k_{1}-k_{2}\right)\left(k_{1}-k_{3}\right)\left(k_{1}-k_{4}\right)} \Omega_{1}+\frac{y_{1} y_{2} y_{3} k_{1} k_{2} k_{3}}{\left(k_{1}-k_{2}\right)\left(k_{2}-k_{3}\right)\left(k_{2}-k_{4}\right)} \Omega_{2} \\
& -\frac{y_{1} y_{2} y_{3} k_{1} k_{2} k_{3}}{\left(k_{1}-k_{3}\right)\left(k_{2}-k_{3}\right)\left(k_{3}-k_{4}\right)} \Omega_{3}+\frac{y_{1} y_{2} y_{3} k_{1} k_{2} k_{3}}{\left(k_{1}-k_{4}\right)\left(k_{2}-k_{4}\right)\left(k_{3}-k_{4}\right)} \Omega_{4}
\end{aligned}
$$

where all components of the transform matrix are identical to those derived from (10).

$$
\begin{aligned}
& A_{1}^{1}=1 \\
& A_{1}^{2}=-\frac{y_{1} k_{1}}{k_{1}-k_{2}}=A_{1}^{1} \frac{k_{1}}{k_{2}-k_{1}} \\
& A_{2}^{2}= \frac{y_{1} k_{1}}{k_{1}-k_{2}}=0-\frac{y_{1} k_{1}}{k_{2}-k_{1}}=c_{2}^{o}-A_{1}^{2} \\
& A_{1}^{3}= \frac{y_{1} y_{2} k_{1} k_{2}}{\left(k_{1}-k_{2}\right)\left(k_{1}-k_{3}\right)}=A_{1}^{2} \frac{y_{2} k_{2}}{k_{3}-k_{1}} \\
& A_{2}^{3}=-\frac{y_{1} y_{2} k_{1} k_{2}}{\left(k_{1}-k_{2}\right)\left(k_{2}-k_{3}\right)}=A_{2}^{2} \frac{y_{2} k_{2}}{k_{3}-k_{2}} \\
& A_{3}^{3}= \frac{y_{1} y_{2} k_{1} k_{2}}{\left(k_{1}-k_{3}\right)\left(k_{2}-k_{3}\right)}=0-\frac{y_{1} y_{2} k_{1} k_{2}}{\left(k_{1}-k_{2}\right)\left(k_{1}-k_{3}\right)}+\frac{y_{1} y_{2} k_{1} k_{2}}{\left(k_{1}-k_{2}\right)\left(k_{2}-k_{3}\right)}=c_{3}^{o}-A_{1}^{3}-A_{2}^{3} \\
& A_{1}^{4}=-\frac{y_{1} y_{2} y_{3} k_{1} k_{2} k_{3}}{\left(k_{1}-k_{2}\right)\left(k_{1}-k_{3}\right)\left(k_{1}-k_{4}\right)}=A_{1}^{3} \frac{y_{3} k_{3}}{k_{4}-k_{1}} \\
& A_{2}^{4}= \frac{y_{1} y_{2} y_{3} k_{1} k_{2} k_{3}}{\left(k_{1}-k_{2}\right)\left(k_{2}-k_{3}\right)\left(k_{2}-k_{4}\right)}=A_{2}^{3} \frac{y_{3} k_{3}}{k_{4}-k_{2}} \\
& A_{3}^{4}=-\frac{y_{1} y_{2} y_{3} k_{1} k_{2} k_{3}}{\left(k_{1}-k_{3}\right)\left(k_{2}-k_{3}\right)\left(k_{3}-k_{4}\right)}=A_{3}^{3} \frac{y_{3} k_{3}}{k_{4}-k_{3}} \\
& A_{4}^{4}= 0+\frac{y_{1} y_{3} k_{1} k_{2} k_{3}}{\left(k_{1}-k_{2}\right)\left(k_{1}-k_{3}\right)\left(k_{1}-k_{4}\right)}-\frac{y_{2} y_{3} k_{1} k_{2} k_{3}}{\left(k_{1}-k_{2}\right)\left(k_{2}-k_{3}\right)\left(k_{2}-k_{4}^{4}\right)}+A_{3}^{4} y_{2} y_{3} k_{1} k_{2} k_{3} \\
&\left(k_{1}-k_{3}\right)\left(k_{2}-k_{3}\right)\left(k_{3}-k_{4}\right)
\end{aligned}
$$

\subsection{Central line concentrations of a BIOCHLOR example}

Aziz et al. [1999] implemented the Sun et al. [1999] solution for multiple first-order decay chains with the same retardation factor in the BIOCHLOR code. To understand what role retardation factors play in the sequentially reactive transport, we use the example of dechlorination in BIOCHLOR.

$$
\begin{array}{cccccccc}
c_{1} & k_{1} & c_{2} & k_{2} & c_{3} & k_{3} & c_{4} & k_{4} \\
(P C E) & \underset{y_{1}}{\longrightarrow} & (T C E) & \underset{y_{2}}{\longrightarrow} & (D C E) & \underset{y_{3}}{\longrightarrow} & (V C) &
\end{array}
$$


where PCE, TCE, DCE, and VC are tetrachloroethylene, trichloroethylene, dichloroethylene, vinyl chloride, respectively. When the system parameters are defined as in Table 2, the central line concentrations are shown in Figure 2. The solid line represents the new solution $c_{i}, \forall i=1,2,3,4$ when unique retardation factors $[7.1,2.8,2.9,1.4]$ are used while the dashed line represents the central-line concentrations, $c_{i}^{\prime}, \forall i=1,2,3,4$, calculated from BIOCHLOR, when the average retardation factor, $\bar{R}=2.9$ is used for every species. Figure 3 shows the steady state concentrations of four species along the central line in the study domain of Domenico [1987]. Initially, $c_{4}$ advances slower than $c_{4}^{\prime}$ (Figure 2), but after steady state is reached, it advances farther (Figure 3).

Table 2. Data set for BIOCHLOR example

\begin{tabular}{|c|c|c|c|}
\hline Velocity & $v$ & 111.7 & ft $y r^{-1}$ \\
\hline Dispersivity & $a$ & 40 & $f t$ \\
\hline Retardation factor 1 & $R_{1}$ & 7.1 & \\
\hline Retardation factor 2 & $R_{2}$ & 2.9 & \\
\hline Retardation factor 3 & $R_{3}$ & 2.8 & \\
\hline Retardation factor 4 & $R_{4}$ & 1.4 & \\
\hline 1st-order reaction rate 1 & $k_{1}$ & 2.0 & $y r^{-1}$ \\
\hline 1st-order reaction rate 2 & $k_{2}$ & 1.0 & $y r^{-1}$ \\
\hline 1st-order reaction rate 3 & $k_{3}$ & 0.7 & $y r^{-1}$ \\
\hline 1st-order reaction rate 4 & $k_{4}$ & 0.4 & $y r^{-1}$ \\
\hline Boundary concentration 1 & $c_{1}^{o}$ & 56.00 & $m g L^{-1}$ \\
\hline Boundary concentration 2 & $c_{2}^{o}$ & 15.80 & $m g L^{-1}$ \\
\hline Boundary concentration 3 & $c_{3}^{0}$ & 98.50 & $m g L^{-1}$ \\
\hline Boundary concentration 4 & $c_{4}^{0}$ & 3.08 & $m g L^{-1}$ \\
\hline Yield coefficient $1 \rightarrow 2$ & $y_{1}$ & 0.79 & \\
\hline Yield coefficient $2 \rightarrow 3$ & $y_{2}$ & 0.74 & \\
\hline Yield coefficient $3 \rightarrow 4$ & $y_{3}$ & 0.64 & \\
\hline Time & $t$ & 1.5 & $y r$ \\
\hline
\end{tabular}




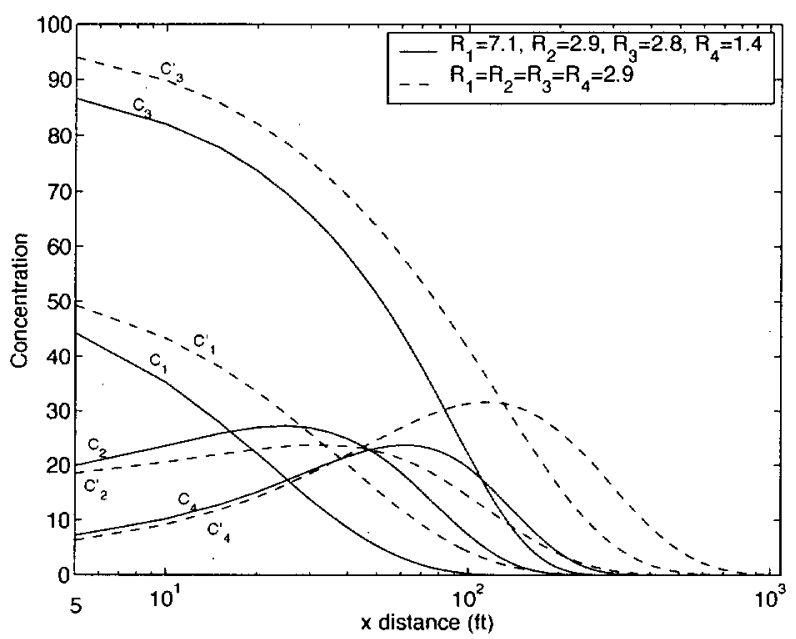

Figure 2. Concentration $c_{i}$ profiles of four species transport calculated using our new solution scheme are compared with central-line concentrations $c_{i}^{\prime}$ calculated from BIOCHLOR.

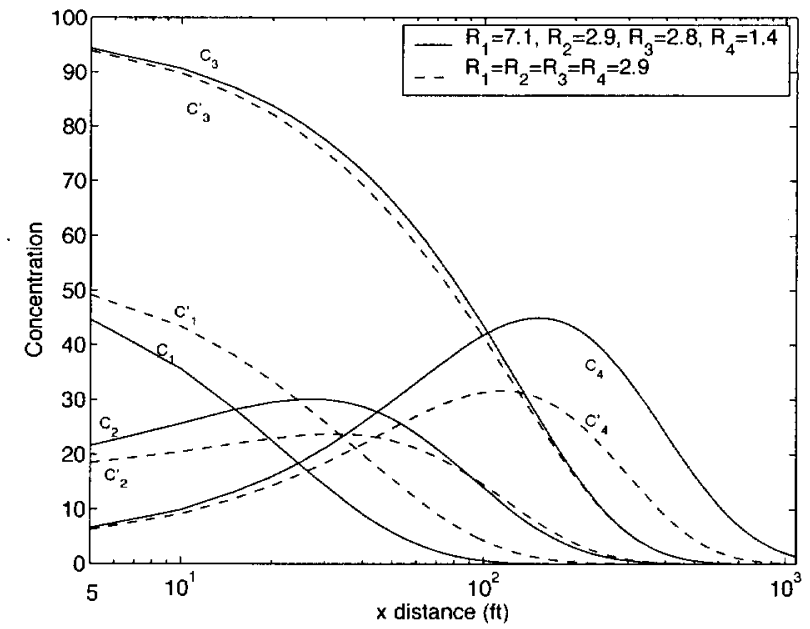

Figure 3. Steady state concentration profiles of four species transport calculated in the study domain of Domenico [1987] are compared to those calculated using our new solution scheme.

\subsection{Extension to Domenico's domain}

Domenico [1987] derived an analytical solution of a single species with first-order decay in three dimensions. Aziz et al. [1999] implemented this solution with the Sun et al. [1999] transform in the BIOCHLOR code. To overcome the restriction of the same retardation factor, here we apply the recursive transform with Domenico's [1987] solution, which can be expressed as the product of lateral distribution factor and longitudinal concentration distribution [Petersen and Sun, 2000]

$$
c(x, y, z)=c^{o} \gamma(x, y, z) \Omega^{\prime}(k)=c^{o} \Omega(k),
$$


where

$$
\gamma(x, y, z)=\frac{1}{4}\left\{\operatorname{erf} \frac{y+Y / 2}{2\left(a_{y} x\right)^{1 / 2}}-\operatorname{erf} \frac{y-Y / 2}{2\left(a_{y} x\right)^{1 / 2}}\right\}\left\{\operatorname{erf} \frac{z+Z / 2}{2\left(a_{z} x\right)^{1 / 2}}-\operatorname{erf} \frac{z-Z / 2}{2\left(a_{z} x\right)^{1 / 2}}\right\}
$$

and $\gamma(0,0,0)=1$. $Y$ and $Z$ are the source dimensions $[L]$, and $a_{x}, a_{y}, a_{z}$ are, respectively, longitudinal, transverse, and vertical dispersivities $[L]$. Since the lateral distribution factor $\gamma$ is a function of spatial variables and dispersivities, it is species independent. Note that $\Omega^{\prime}$ here is equivalent to $\Omega$ in (13) in the one-dimensional system. The basic solution for species $i$ in three-dimensional Domenico's domain is redefined as

$$
\Omega_{i}=\frac{\gamma}{2} \exp \left(\alpha_{i} x\right) \operatorname{erfc}\left(\beta_{i}\right) \quad \forall i=1,2, \cdots, n
$$

Correspondingly, the first derivative of $\Omega$ is

$$
\frac{\partial \Omega_{i}}{\partial t}=\frac{\gamma}{4 \sqrt{D_{i} \pi}} \exp \left(\alpha_{i} x-\beta_{i}^{2}\right)\left[\frac{x}{t^{3 / 2}}+\sqrt{\frac{v_{i}^{2}+4 k_{i} D_{i}}{t}}\right] \quad \forall i=1,2, \cdots, n .
$$

Using the same system parameters in Table 2, the concentration profiles of four species, when $t=15 y \mathrm{r}$ and $z=0$, are shown in Figure 4. Additional parameters are given as $a_{x}=40, a_{y}=10$, $a_{z}=10$. Figure 4 also shows the comparison of concentration profiles (left column) derived from the new solution scheme and those (right column) calculated from BIOCHLOR. 

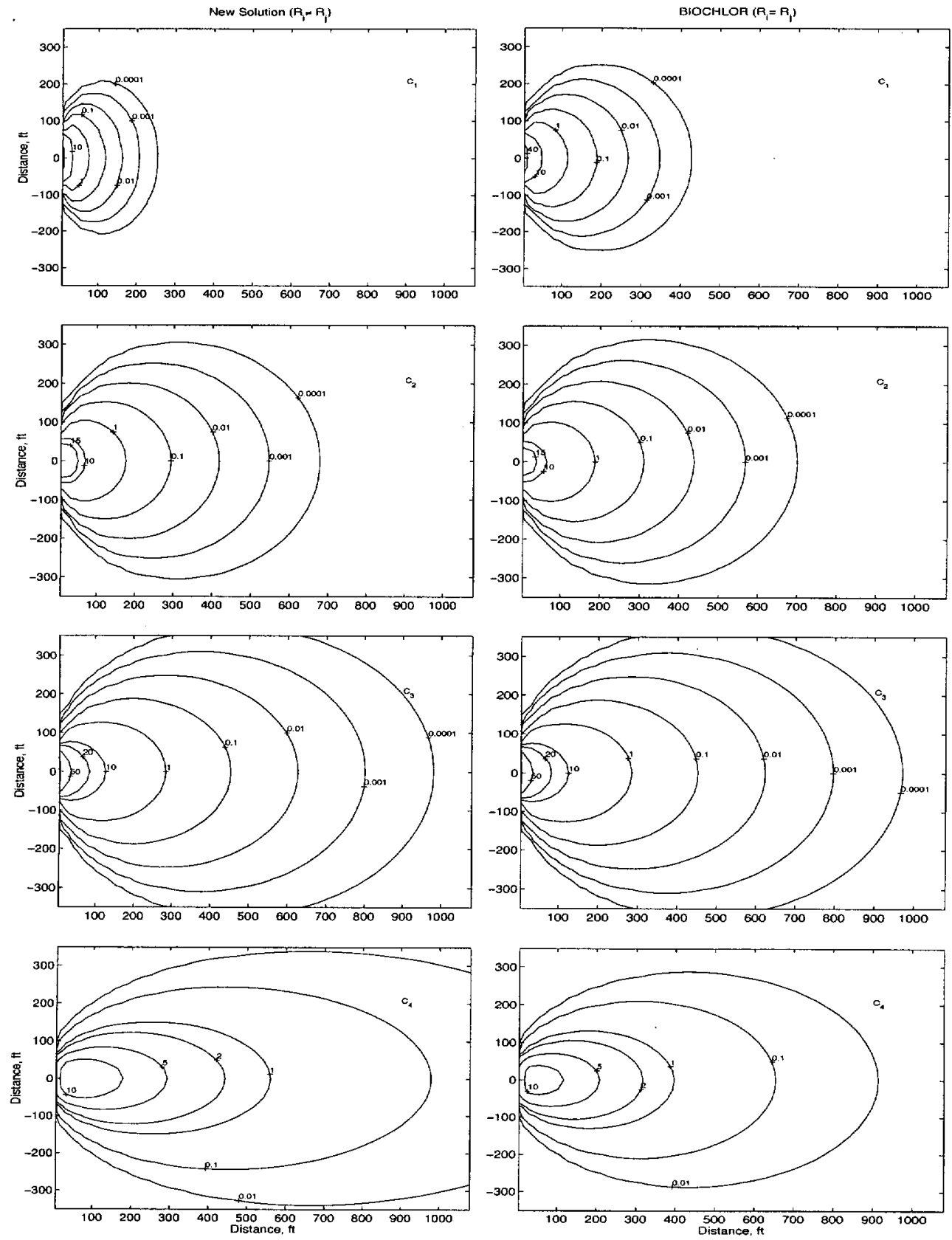

Figure 4. Concentration profiles of four species transport in Domenico's domain. Four contour plots in the left column are derived from the new solution scheme, $R=[7.1,2.9,2.8,1.4]$, and four plots in the right column are calculated from BIOCHLOR, $\bar{R}=2.9$. 


\subsection{Sensitivity Analysis}

The effect of linear sorption on the mass transport of a single species has been extensively studied [Bear, 1979], To estimate the effect of retardation factors of parent species on the daughter species and understand the behavior of the sequentially reactive transport systems with different retardation factors, a sensitivity analysis is conducted using Lunn et al.'s [1996] model and the new solution. By changing $R_{1}, R_{2}, R_{3}$, respectively, and fixing all other system parameters, the concentration profiles of the end product are shown in Figure 5. The thick solid line represents the base case when $R_{1}=2, R_{2}=1, R_{3}=1$ as shown in Figure 1 . Higher values of $R_{1}$ (grandparent), $R_{2}$ (parent), and $R_{3}$ (itself) make the transport of $c_{3}$ slower, but higher values of $R_{1}$ and $R_{2}$ make the concentration higher upstream and lower downstream while the higher value of $R_{3}$ makes the concentration of $c_{3}$ lower anywhere. 

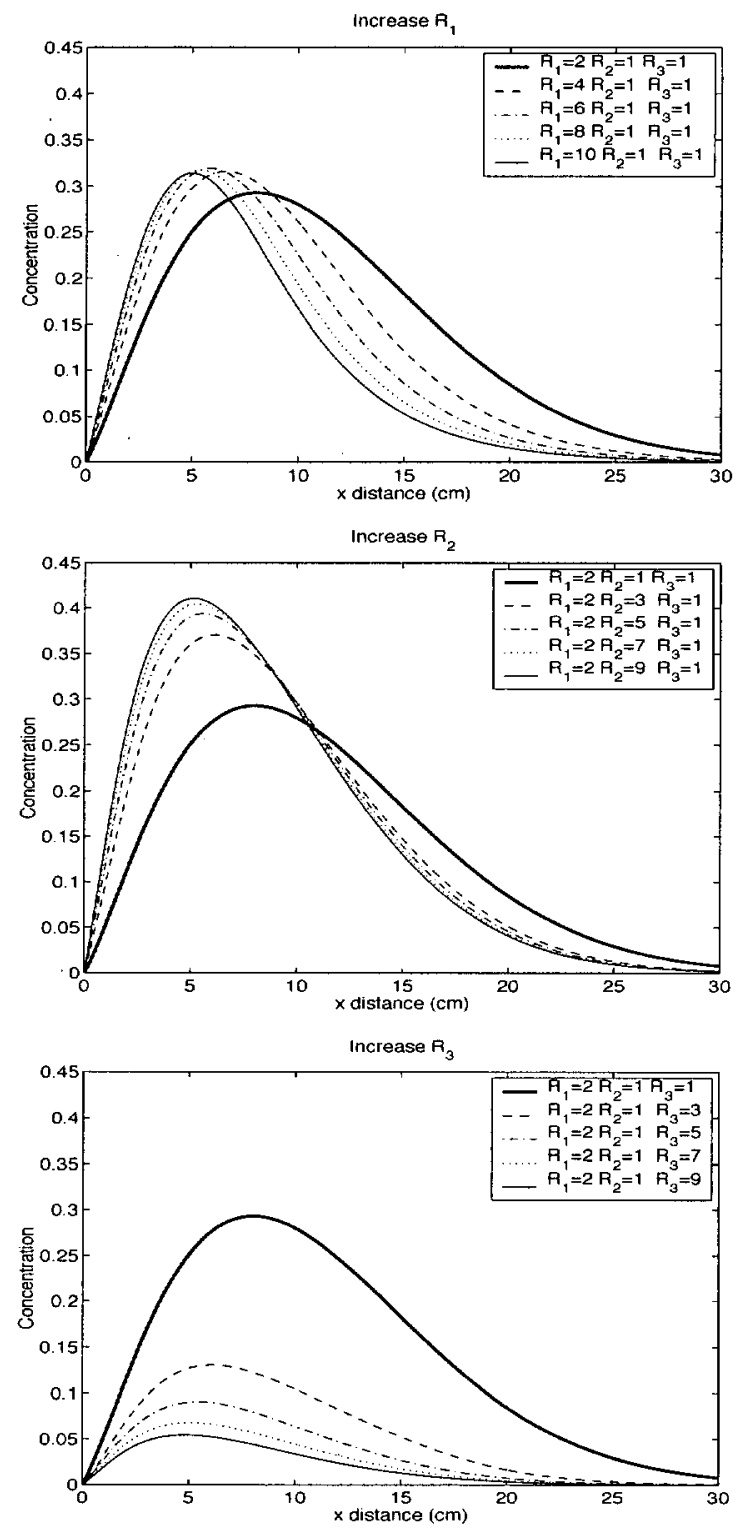

Figure 5. Concentration profiles of species 3 in Lunn et al.s' [1996] model after $200 \mathrm{~h}$.

\section{Conclusions}

A solution scheme has been developed in the time domain for deriving analytical solutions of sequentially reactive transport systems with different retardation factors. To avoid the difficulty inherent in using inverse Laplace transforms, previously published analytical solutions for a single transporting species in the time domain are used as fundamental solutions. Since the partial differential equations are linear in species concentrations, a daughter species concentration can be expressed as a linear function of those fundamental solutions. The solution scheme accounts for stoichiometric 
yield coefficients. One unit of parent species unnecessarily produces one unit of daughter species. It has been demonstrated that Sun et al. [1999] transform with the same retardation assumption is a typical scenario in the recursive transform presented in this paper. The new solution derived using the solution scheme matches Lunn et al. [1996] for three species transport in a one-dimensional column.

This work was performed under the auspices of the U. S. Department of Energy by the University of California, Lawrence Livermore National Laboratory under Contract No. W-7405-Eng-48.

\section{Acknowledgements}

The authors wish to thank Jacob Bear at Technion-Israel Institute of Technology for stimulating discussions and an initial review for the manuscript.

\section{References}

Aziz, C. E., C. J. Newell, G. R. Gonzales, P. E. Haas, T. P. Clement, Y. Sun, BIOCHLOR - Natural attenuation decision support system, Beta version 1.0, User Manual, Subsurface Protection and Remediation Division, National Risk Management Research Laboratory, Ada, Oklahoma 74820, 1999.

Bauer, P., S. Attinger, W. Kinzelbach, Transport of a decay chain in homogenous porous media: analytical solution, Journal of Contaminant Hydrology 49, 217-239, 2001.

Bear, J., Groundwater hydraulics, McGraw-Hill, New York; 1979.

Clement, T. P., Y. Sun, B. S. Hooker, B. S., and J. N. Petersen, Modeling multi-species reactive transport in groundwater aquifers, Groundwater Monitoring and Remediation 18(2), 79-92, 1998.

Domenico, P. A., An analytical model for multidimensional transport of a decaying contaminant species, Journal of Hydrology 91, 49-58, 1987.

Eykholt, G. R., L. Li Fate and transport of species in a linear reaction network with different retardation coefficients Journal of Contaminant Hydrology 46, 163-185, 2000.

Lunn, M., R. J. Lunn, R. Mackay, Determining analytic solutions of multiple species contaminant transport with sorption and decay, Journal of Hydrology 180, 195-210, 1996.

Press, W. H., S. A. Teukolsky, W. T. Vetterling, B. P. Flannery, Numerical Recipes in C, Cambridge University Press, Cambridge, 1996.

Petersen, J. N., Y. Sun, An analytical solution evaluating steady-state plumes of sequentially reactive contaminants, Transport in Porous Media 41, 287-303, 2000.

Sun, Y., Petersen, J. N., Clement, T. P., and R. S. Skeen, R. S., Development of analytical Solutions for multi-species transport with serial and parallel reactions, Water Resources Research $\mathbf{3 5}(\mathbf{1})$, 185-190, 1999.

van Genuchten, M. Th., Convective-dispersive transport of solutes involved in sequential first-order decay reactions, Computers and Geosciences 11(2), 129-147, 1985.

\footnotetext{
${ }^{1}$ Lawrence Livermore National Laboratory, Livermore, CA 94550, USA.

${ }^{2}$ Weiss Associates, Emeryville, CA 94608, USA.

${ }^{3}$ California State University, Hayward, CA 94542 , USA.
}

This preprint was prepared with Yunwei Sun's LAIEX macros v4. File retard formatted August $23,2001$. 